\title{
Calcul de température des cuves dans les réacteurs à neutrons rapides de type intégré
}

\author{
par $\mathbf{M}^{\mathrm{me}}$ 0. Daubert et M. J.-F. Malherbe \\ Laboratoire National d'Hydraulique, \\ E.D.F., Chatou
}

\section{I - Les problèmes de thermohydraulique dans les réacteurs à neutrons rapides du type intégré}

\section{1 - Introduction}

Les températures des cuves des réacteurs à neutrons rapides intégrés sont étroitement liées à la géométrie du bloc-pile. C'est, en effet, l'architecture des cuves elles-mêmes et des composants qui détermine, dans leurs grandes lignes, les écoulements du fluide caloporteur. Les écoulements déterminent ensuite le champ des températures avec des interactions possibles. Une fois les températures évaluées, c'est au mécanicien d'estimer si les contraintes qu'elles induisent dans les structures sont admissibles.

Les propos qui vont suivre s'inspirent essentiellement des études thermohydrauliques relatives à l'architecture particulière du réacteur Phénix, prototype français de la filière rapide intégrée. On accordera cependant à ces propos, un certain caractère de généralité, car les réacteurs de la filière, de puissance plus grande et actuellement à l'étude, constituent dans une large mesure une extrapolation du prototype.

\section{I.2 - Description sommaire de l'hydraulique de Phénix}

Le bloc-pile de celui-ci a déjà été abondamment décrit, et l'on rappellera simplement que, vu par l'hydraulicien, il est conçu de la manière suivante (cf. fig. 1):

- Dans une cuve, dite principale, sont intégrés le cœur du réacteur et l'ensemble des structures canalisant le fuide caloporteur primaire, en l'occurrence du sodium liquide (on désigne sous le nom de primaire le sodium qui passe au cœur de la réaction nucléaire pour en extraire les calories). La cuve principale est suspendue par des attentes à une dalle formant toit et est porteuse de tout ce qu'elle contient.

- A l'intérieur de la cuve principale, se trouve une cuve primaire collectant le sodium chaud issu du cœur (en moyenne $560^{\circ} \mathrm{C}$ ). Le sodium chaud passe ensuite dans des échangeurs dits intermédiaires. Il y cède sa chaleur à un sodium secondaire qui emporte les calories hors du blocpile, à travers le toit, vers des générateurs de vapeur. A la sortie des échangeurs intermédiaires, le sodium primaire redevenu froid $\left(400{ }^{\circ} \mathrm{C}\right)$ est collecté dans l'espace situé entre les deux cuves, espace appelé intercuve.

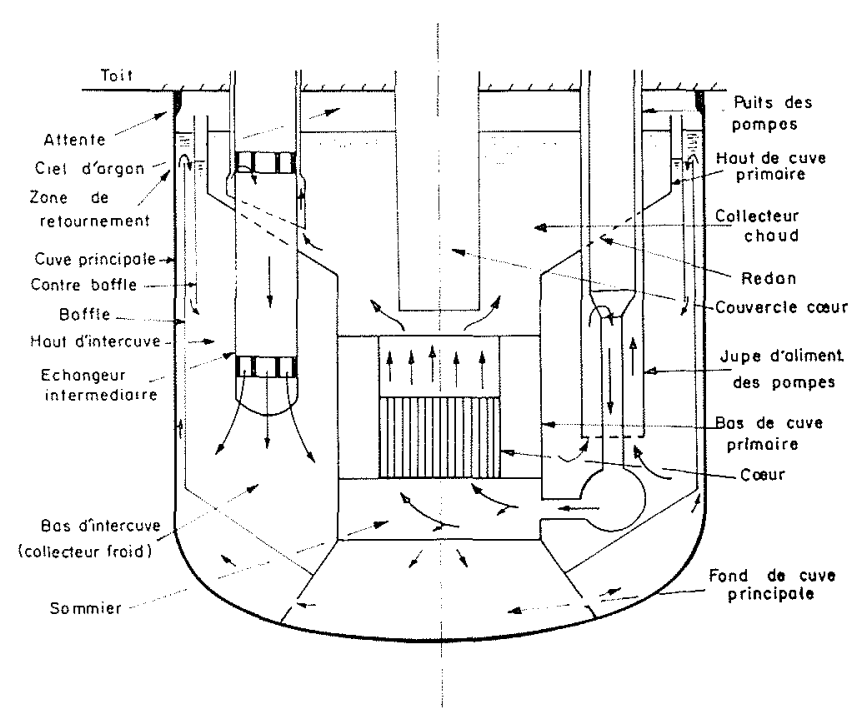

1/ Schèma de principe du réacteur Phénix. Coupe par pompe-échangeur 
Il est donc nécessaire que les échangeurs puissent baigner dans chacun des deux collecteurs pour en assurer la liaison. C'est la raison pour laquelle la cuve primaire comporte, en sa partie haute, une virole de diamètre supérieur à celui qu'elle présente en sa partie basse. Les deux viroles, haute et basse, sont solidarisées par un redan presque horizontal à travers lequel passent les échangeurs.

- Enfin, le sodium du collecteur froid est aspiré par des pompes plongeant dans l'intercuve et refoulé vers le cœur. Les corps des pompes traversent le redan et le toit comme les échangeurs intermédiaires, mais ici uniquement pour des raisons d'encombrement et d'accessibilité. Les composants sont groupés par deux échangeurs entourant une pompe. Le nombre de ces groupes sont de trois dans Phénix et disposés de manière à assurer une bonne symétrie circonférentielle au réacteur.

L'existence de deux collecteurs de volume suffisant est utile en cas de chocs thermiques intenses : chocs froids dans la cuve primaire par chute des barres de contrôle dans le cour et arrêt de son fonctionnement, chocs chauds dans l'intercuve en cas de panne dans les circuits secondaires et arrêt de l'extraction des calories dans les échangeurs intermédiaires. Les masses contenues dans les collecteurs ont alors un rôle de volant thermique et amortissent les variations brusques de températures imposées aux structures.

\section{3 - Nature des problèmes posés}

Ils sont liés à la proximité des deux collecteurs chaud et froid, qui est inhérente à la nature intégrée du réacteur. L'excellente conductivité du sodium entraîne, alors, des transferts importants de puissance d'une zone à l'autre et de forts gradients sur les structures, en particulier sur les curves. Ces transferts se produisent, non seulement sur les parois léchées par les convections forcées, mais aussi là où les convections forcées pénètrent peu, car les propriétés physiques du sodium le rendent apte à développer de fortes convections naturelles: par exemple, une plaque plane verticale, de hauteur de $2 \mathrm{~m}$ et plongée dans un bain de sodium à $30^{\circ} \mathrm{C}$ plus froid sera le moteur d'une convection pouvant atteindre $50 \mathrm{~cm} / \mathrm{s}$, soit plus que la vitesse du sodium à la sortie des échangeurs de Phénix (environ $30 \mathrm{~cm} / \mathrm{s}$ ). Outre les gradients de température sur les structures, les transferts internes ont aussi pour conséquence une dégradation du point chaud et une perte sur le rendement du réacteur.

Etant donné les considérations précédentes, les problèmes les plus aigus qui se posaient alors pour les cuves résidaient, d'après les mécaniciens et métallurgistes, essentiellement dans les points suivants:

\subsection{1 - En régime établi, nominal ou partiel}

Problème 1. - La température de la cuve principale: elle doit rester en tout point inférieure à une température dépassant peu $500{ }^{\circ} \mathrm{C}$ (alors que celle du collecteur chaud, tout proche, est de $560^{\circ} \mathrm{C}$ ) et n'être soumise à aucun gradient longitudinal. C'est pourquoi un circuit de refroidissement de la cuve principale a été établi : ce circuit utilise le débit de fuite aux pieds des assemblages constituant le cœur. Le sodium est guidé, le long de la cuve principale, à

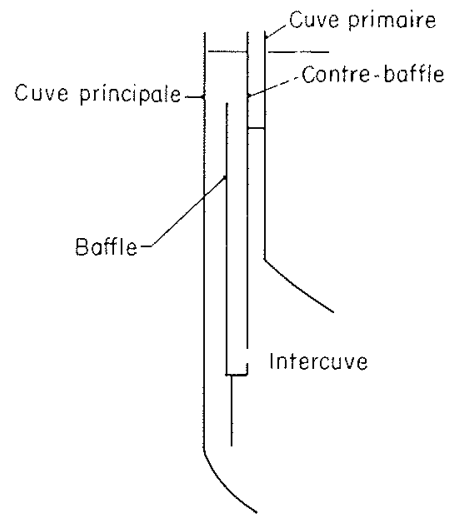

l'intérieur d'un baffle cylindrique et redescend dans l'intercuve guidé par un contrebaffie. Des pertes de charge, égales à celles des échangeurs, sont créées dans le contrebaffle pour que la surface libre dans le circuit se cale au même niveau que dans la cuve primaire et soit donc fixe, quels que soient les débits (lorsque les débits varient, c'est en effet la surface libre de l'intercuve, la plus faible, qui encaisse les variations en raison de la conservation des volumes). En régimes froids, les niveaux sont au plus bas par suite de la contraction du sodium. On arase le baftle légèrement en-dessous pour qu'il n'y ait pas déversement vers la redescente dans le contrebaffle, avec les risques d'entraînement d'argon que cela comporte. Aux régimes chauds, il y a expansion du sodium et les niveaux libres se placent à environ un demi-mètre au-dessus de l'arête supérieure du baffle. L'expérience montre que, dans de tels cas, les débits de l'ordre de celui utilisé dans le circuit restent concentrés sur quelques centimètres au-dessus du seuil sur lequel ils se retournent. Il existe donc, aux régimes chauds, une zone dite de retournement, stagnante et soumise à l'influence du collecteur chaud tout proche. En fin de compte, le problème était, d'une part de vérifier l'efficacité du circuit de refroidissement jusqu'à la cote du retournement et au besoin de contribuer à le dimensionner; d'autre part, d'évaluer l'influence du collecteur chaud dans la zone de retournement. Ce dernier point était surtout nécessaire à régime partiel chaud, le canal annulaire entre le contrebaffle et la cuve primaire étant rempli de sodium, meilleur conducteur que l'argon du ciel qui y prend place au régime nominal.

Problème 2. - Les gradients de température à travers et le long de la cuve primaire: c'est le redan qui est le plus sensible en raicon de sa position en porte à faux, et particulièrement les deux tores qui le raccordent aux deux viroles cylindriques. La position haute du redan aménage, dans l'intercuve, une zone peu soumise aux convections forcées induites entre la fenêtre de sortie des échangeurs et la jupe d'aspiration des pompes. En effet, il a été constaté, sur un modèle physique d'échangeur construit au Laboratoire National d'Hydraulique (L.N.H.), que le jet issu de la fenêtre de sortie avait une tendance naturelle à plonger vers le bas. Cette tendance est encore renforcée par l'aspiration des pompes, dont les jupes d'alimentation ont leur extrémité au bas de l'intercuve. Au-dessus de la cote supérieure des fenètres de sortie des échangeurs, peuvent se développer des convections naturelles le long de la paroi chaude de la cuve primaire. Dans ces conditions, le haut d'intercuve se maintient à une température inter- 
médiaire, constituant une zone tampon pour le redan entre la partie hatite de la cuve primaire et le bas d'intercuve. Ceci atténue les gradients transversaux à travers le redan. Par contre, les convections naturelles induisent une stratification génératrice de gradients longitudinaux. C'est pour protéger la cuve principale contre les températures chaudes du haut d'intercuve, et contre sa stratification, qu'a été constitué le circuit des baffles. Réciproquement, la faible convection forcée, créée par le rejet de ce circuit dans le haut d'intercuve, permettait d'espérer la destruction d'une stratification trop brutale. Il s'agissait donc de régler le rejet à une cote, ni trop haute pour que les températures restent assez chaudes sous le redan, ni trop basse pour que son rôle de déstratification soit conservé et que le haut d'interouve soit le plus homogène possible. Il restait, dans cette solution, à s'assurer des gradients transversaux imposés à la virole cylindrique de cuve primaire, au droit du cour. Ces températures ont été évaluées, par le S.E.M.T.R. de Cadarache, à l'occasion de l'étude du refroidissement de la protection neutronique latérale du cour.

\subsection{2 - En régimes transitoires}

On distingue, d'une part, les régimes transitoires rapides comme les chocs thermiques déjà évoqués, d'autre part, les régimes transitoires lents comme les démarrages ou comme les prooédures de refroidissement général du réacteur pour intervention.

- En ce qui concerne les chocs, ils sont d'autant plus amortis que les collecteurs concernés sont mieux brassés dans leur ensemble. Les effets gravitaires, induits dans les écoulements par les différences de température, ne sont alors pas à négliger. En cas d'arrivée de sodium froid dans le collecteur chaud, la position haute des cloches d'échangeur assure de bonnes circulations verticales, malgré les différences de masse volumique. Ceci a été vérifié sur un modèle, réduit au quart, du collecteur chaud réalisé par SOGREAH et fonctionnant en eau salée et eau douce (on peut en effet négliger les phénomènes de conduction dans le cas des chocs et procéder pour leur étude par des expériences non en similitude à ce point de vue).

En cas de choc chaud dans l'intercuve, la partie basse reste brassée par les fortes convections forcées dont elle est le siège, et contribue à amortir le choc. Les effets de gravité tendent, de plus, à faire envahir la partie haute par le sodium chaud sortant des échangeurs. C'est ainsi que la zone tampon joue le rôle de volant thermique qui lui était a priori assigné.

- Pour les régimes transitoires proprement dits, le problème le plus aigu concerne les températures dans la zone des attentes: l'inertie thermique du toit est, en effet, très grande et ne suit pas l'évolution des températures dans la zone de retournement. D'où des gradients longitudinaux importants imposés aux attentes. Pour le calcul de celles-ci, il fallait donc connaître l'évolution des températures dans la zone du retournement, ce qui rejoint les préoccupations déjà évoquées au sujet des températures imposées à l'ensemble de la cuve principale en régimes établis (problème 1). Le même ensemble de problèmes qu'en régimes établis se pose d'ailleurs en régimes transitoires, avec en plus la nécessité de vérifier qu'au cours de ceux-ci, les écarts de température de part et d'autre des structures ne s'inversent pas, en entraînant des inversions de contrainte.

\section{II - Les moyens d'étude Nature des calculs et justification}

\section{II.1 - Nécessité de l'étude par les calculs}

L'analyse précédente a montré la nécessité d'études pour déterminer les températures à l'intérieur des baffles, dans la zone de retournement et dans le haut d'intercuve. Mis à part le circuit des baffles, il s'agit de zones où se développent des convections naturelles car elles sont peu intéressées par les convections forcées, sauf en leur limite inférieure. L.es méthodes d'approche, pour les problèmes de convection mixte dans les métaux liquides, ont fait l'objet d'une analyse détaillée dont on trouvera les éléments dans le document cité en référence [1]. On peut tenter de résumer cette analyse en disant que l'étude par des modèles physiques est impossible, non seulement pour des raisons de maîtrise des conditions aux limites thermiques (puissances échangées à travers les parois), mais surtout pour des raisons de similitude : près des parois, siège des phénomènes essentiels étudiés, il est nécessaire de respecter du point de vue mouvement le nombre de Grashof (carré du rapport entre le nombre de Reynolds et le nombre de Froude densimétrique). Les mouvements sont liés, par les effets gravitaires, aux échanges de chaleur, pour lesquels il faut conserver le nombre de Prandtl ou le nombre de Rayleigh (produit d'un nombre de Grashof par le nombre de Prandtl). Les échanges par entraînement, à la limite inférieure de l'intercuve ou de la zone de retournement, imposent le respect du nombre de Peclet et de Reynolds. Les conditions thermiques imposent à l'expérimentateur d'utiliser un métal liquide, et la faible viscosité des métaux liquides impose alors pratiquement l'échelle 1, pour respecter les transferts de quantité de mouvement.

Devant ces difficultés on a eu recours à des calculs, mais on ne doit pas ignorer les limites de ceux-ci. En particulier, la puissance des moyens de calcul ne permet pas de dépasser le cadre d'une géométrie simple bidimensionnelle.

\section{II.2 - Expériences ayant guidé les calculs}

L'expérience n'est pas exclue du travail de réflexion préalable aux calculs et peut conduire fort utilement ceux-ci. Pour la zone de retournement, une maquette en fluide homogène a montré que le débit des baffles passait seulement sur quelques centimètres au-dessus du seuil de retournement. Ce résultat a été retrouvé par le calcul tenant compte de l'échauffement de la zone située au-dessus de ce seuil.

De même, l'hypothèse de la ségrégation de l'intercuve en deux parties était étayée par l'observation, sur le modèle d'échangeur, de la forme des jets de sortie, ainsi que sur la maquette en fluide homogène SOGREAH, par la mesure des vitesses de recirculation induites par ces jets dans le haut d'intercuve. La comparaison des vitesses de recirculation avec celles obtenues par les calculs classiques de couche limite dans les convections naturelles, ont montré que celles-ci seraient nettement prépondérantes et bloqueraient par effet de densité la recirculation des jets. Une maquette L.N.H., au $1 / 10$, du réacteur a confirmé différemment cette tendance à la ségrégation: le sodium froid, 
issu des échangeurs, était représenté par de l'eau salée et les échanges de chaleur, à travers les parois de la cuve primaire, étaient simulés qualitativement par un apport d'eau douce en haut de l'intercuve. Un faible débit d'eau doutce suffisait à maintenir la zone de transition juste au-dessus de la fenêtre des échangeurs (primitivement ce débit de simulation était d'ailleurs réel, car des fuites à la traversée du redan par les échangeurs étaient envisagées). Cette expérience répétée en eau chaude au S.E.M.T.R. de Cadarache, a confirmé les résultats et, en particulier, un bas d'intercuve correctement brassé et une tendance nette à l'horizontabilité des isothermes. Ceci a écarté la crainte de gradients de température circonférentiels.

Les effets dynamiques gravitaires, dus aux différences de densité, étaient représentés dans les expériences qui viennent d'être évoquées, mais le facteur important de transfert qu'est, dans les métaux liquides, la conduction, ou diffusion moléculaire, n'était par contre évidemment pas en similitude. Cependant, les indications données par les expériences ont permis de justifier une schématisation bidimensionnelle pour des calculs qui, eux, peuvent prendre en compte la conduction. En effet, dans l'intercuve, la partie basse est soumise à des convections forcées de caractère nettement tridimensionnel. Mais la partie haute est le domaine des convections naturelles et celles-ci suivent la cuve primaire, en présentant donc une symétrie circonférentielle, seulement perturbée sous le redan par les composants. Cette symétrie est plus nette dans la zone de retournement au-dessus du baffle.

Des calouls bidimensionnels ont donc été entrepris, dans lesquels sont résolues les équations de Navier-Stokes couplées à l'équation de la chaleur par les termes de gravité.

\section{II.3 - Stratégie menée dans la conduite des calculs. Utilité d'un schéma du bloc-pile}

La détermination de la température de la cuve principale passe, nécessairement, par une étude thermique et hydraulique du débit guidé par les baffles le long de celle-ci. Mais la température de ce circuit dépend de celle qui règne dans l'intercuve.

Il apparaît, d'une manière générale, difficile de dissocier les diverses zones du réacteur les unes des autres. D'où la conception d'un modèle d'ensemble ponctualisant ces zones, et rendant compte de leur température moyenne en fonction de leurs échanges mutuels. Un modèle de ce type, étendu à la centrale tout entière, est toujours constitué pour les études de fonctionnement. Mais la complexité déjà grande de la chaîne éudiée (depuis le cœur jusqu'aux turbines) n'autorise pas ce modèle à détailler le bloc-pile dans l'optique du calcul des structures. Le modèle ponctuel, particulier au bloc-pile, s'y inscrit donc comme un effet loupe: il précise la dégradation du point chaud à l'entrée des échangeurs, sous l'action des fuites thermiques à travers la cuve primaire, et il précise surtout les températures moyennes dans chaque zone, en fonction de l'état ou de l'évolution des paramètres de fonctionnement qui sont aux bornes du bloc-pile:

- la température chaude à la sortie du cœur;

- la température froide à la sortie des échangeurs.

Le schéma, auquel on aboutit ainsi, représente bien les températures dans les zones brassées par les convections forcées. Mais il est dans l'incapacité de fournir les valeurs des gradients de température dans les zones soumises aux convections naturelles et dont la stratification n'est évidemment pas traduite. La valeur des températures moyennes permet cependant aux mécaniciens de détecter les risques de gradients dangereux suivant l'état du réacteur. On peut alors mettre en ceuvre des calculs bidimensionnels, dont les conditions aux limites sont données par le modèle d'ensemble, et qui précisent les répartitions locales de température. C'est ce qui a été fait :

$1^{\circ}$ pour la zone de l'intercuve comprise entre le redan et le jet des échangeurs;

$2^{\circ}$ pour le sodium stagnant au-dessus du retournement du circuit de refroidissement aux régimes établis, nominal et partiel.

\section{III - Calculs globaux du comportement général du bloc-pile}

On distingue dans le bloc-pile les zones suivantes:

- le fond de la cuve principale, collecteur du débit de fuite aux pieds des assomblages;

- le bas dintercuve;

- le haut d'intercuve;

- le cœur;

- la cuve primaire, ou collecteur chaud;

- la zone des baffles;

- les échangeurs;

- les pompes.

\section{III.1 - Détail des échanges pris en compte}

On trouvera, schématisés sur la figure 2, les types d'échanges aurixquels ces zones sont soumises. Les transferts par convection forcée n'offrent aucune difficulté à être traduits. Pour les transferts par convection naturelle, on utilise les coefficients d'échange moyen expérimentaux donnés par la littérature (cf. réf. [2]).

- En ce qui concerne la virole inférieure de la cuve primaire et la partie du contrebaffle baignant dans la zone de retournement, on a utilisé, vu leur grand diamètre, les formules de plaque plane verticale: elles conduisent à un coefficient d'échange:

$$
h==10^{3} \Delta T^{1 / 3} \mathrm{~W} / \mathrm{m}^{2}{ }^{\circ} \mathrm{C}
$$

où $\Delta T$ représente l'écart inconnu des températures à travers la couche limite de convection naturelle (c'est-à-dire l'écart entre la température de la paroi et la température moyenne de la zone concernée par la convection naturelle).

- En ce qui concerne le redan, considéré comme une plaque plane inclinée, le coefficient adopté est:

$$
\begin{gathered}
h=(\sin a)^{1 / 4} \times 10^{3} \Delta T^{1 / 3} \mathrm{~W} / \mathrm{m}^{2}{ }^{\circ} \mathrm{C} \\
=0.77 \times 10^{3} \Delta T^{1 / 3} \mathrm{~W} / \mathrm{m}^{2}{ }^{\circ} \mathrm{C} \\
(a=\text { angle d'inclinaison sur l'horizontale }) .
\end{gathered}
$$

- Les échanges entre le haut d'intercuve et l'intérieur de la jupe des pompes, cause de dégradation de la température froide à l'entrée du cœur, sont représentés par le coefficient suivant, tiré de la littérature: 


$$
h=\lambda / 2 e\left(4,82+0,0185 \mathrm{Pe}^{0,527}\right)
$$

où $\lambda=$ conductivité du sodium;

$e=$ largeur de l'espace annulaire entre la jupe des pompes et la tuyauterie de refoulement de celles-ci;

$\mathrm{Pe}=$ nombre de Peclet de l'écoulement forcé dans l'espace annulaire.

La formule n'est valable que pour les nombres de Peclet élevés.

- En ce qui concerne la zone des baffles, on a pu détailler l'évolution verticale de la température, ceci par la mise en œuvre d'un calcul classique du type échangeurs. Les échanges entre les parois et les écoulements sont ceux correspondant aux faibles nombres de Péclet soit:

$$
h=7 \lambda / 2 e
$$

(e représente ici la largeur de l'espace entre les parois du circuit).

Le modèle donne donc les températures tout le long du circuit et, en particulier, les températures le long de la cuve principale. Les fuites à travers celle-ci ont été prises en compte par le biais d'un coefficient d'échange calculé par ailleurs.

Pour le calcul des régimes transitoires, les températures dans les baffles ont été considérées comme issues d'une succession d'états d'équilibres. C'est-à-đire que leur réponse aux variations des températures, dans les autres zones, ont été considérées comme instantanées (en effet le temps de parcours du circuit est de quelques minutes et les régimes transitoires sont à évolution lente sur plusieurs heures). Cette partie du modèle concernant les baffles a contribué à leur dimensionnement, ainsi qu'à la détermination du débit de fuite suffisant.

\section{III.2 - Mise en équation. Exemple de résultałs}

Chacun des coefficients d'échanges décrits ci-dessus, est composé avec la résistance des parois. La mise en équation se fait ensuite simplement par l'écriture des bilans thermiques, zone par zone, et conduit à la résolution d'un système non linéaire. Pour le calcul des régimes transitoires, on ajoute au système les termes d'inertie thermiques qui comprennent :

- les volumes de sodium de chacun des collecteurs;

- les masses d'acier contenues.

Les points d'entrée des calculs sont comme déjà dit les températures à la sortie du cœur et des échangeurs.

On trouvera des exemples de résultats sur les figures suivantes :

- figure 3: carte des températures pour un régime établi;

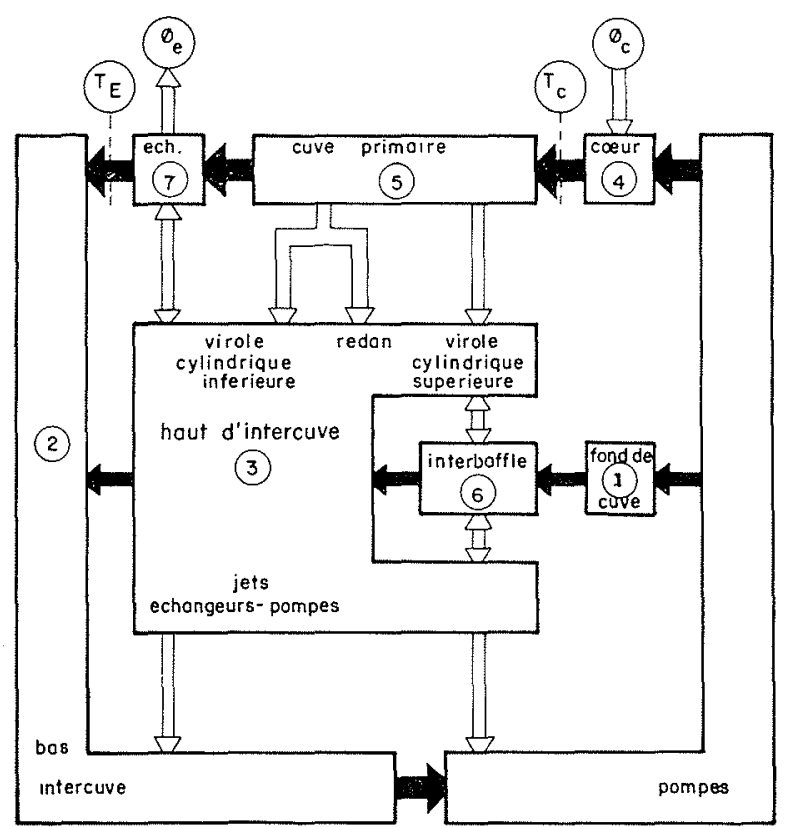
Les flèches horizontales norres indiquent un tronsfert por
convection forcée
$\square$ Les flèches verticales blonches indiquent un tronsfert par conduction
por convection noturelle ou por turbulence

2/ Schéma descriptif des échanges dans le réacteur.

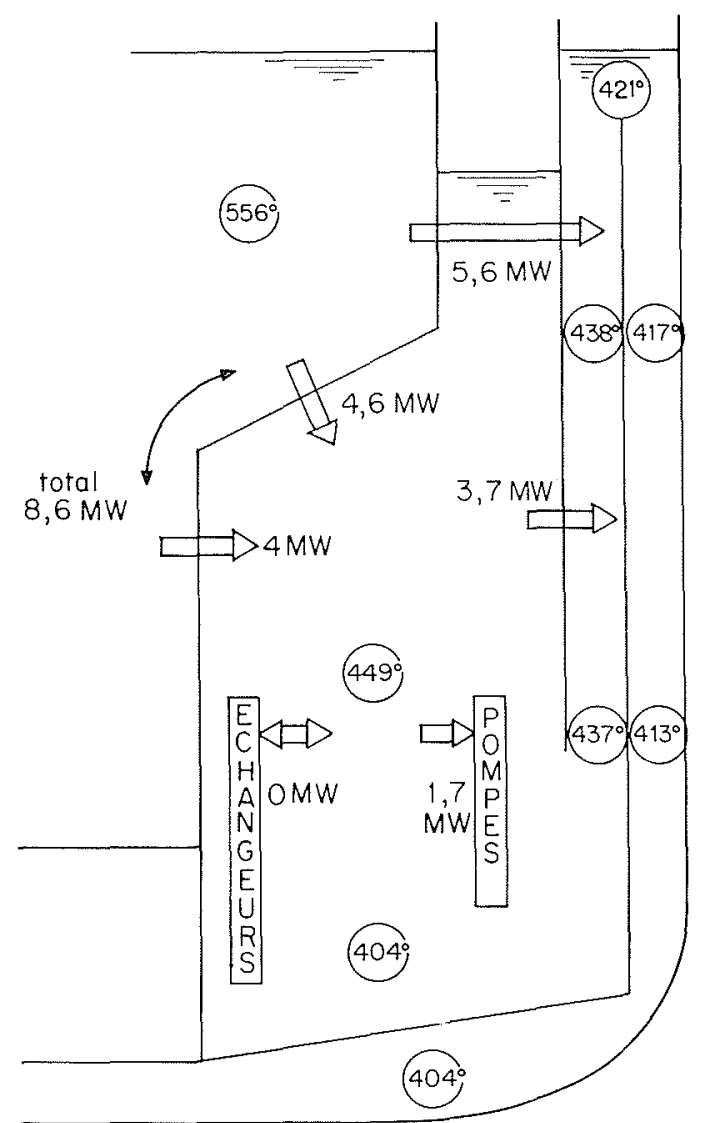

Température chaude (sortie coeur) $T_{C}=560^{\circ} \mathrm{C}$

Température froide (sortie échangeur) $T_{E}=400^{\circ} \mathrm{C}$
3/ Carte des températures et des puissances échangées par conduction et convection naturelle en régime établi $A_{0}$. 


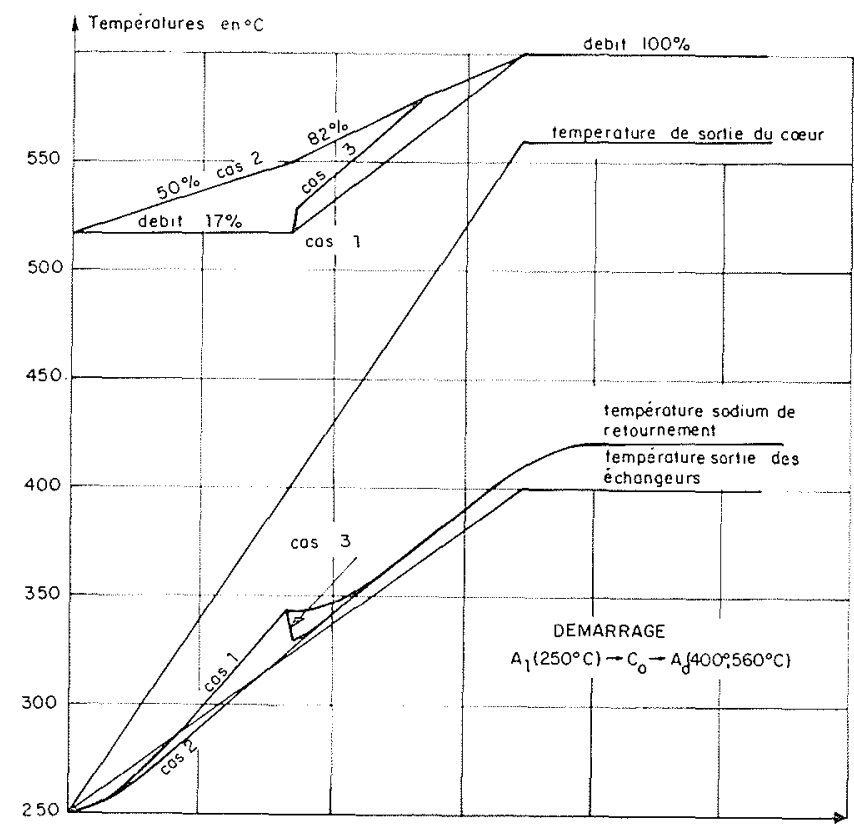

$4 /$

- figure 4: évolution des températures en bout de circuit des baffles pour un cas de démarrage;

- figure 5: même chose pour un cas d'arrêt.

\section{IV - Câlculs bidimensionnels}

Dans tous les cas où ni les expériences, ni les calculs globaux, ne peuvent répondre aux problèmes posés, en particulier lorsqu'on présume l'existence d'une stratification, on est tenté d'utiliser des moyens de calcul qui, bien que très imparfaits, permettent, à la fois, de différencier les points de l'espace et de tenir compte des propriétés thermiques du sodium liquide : ce sont les modèles bidimensionnels d'écoulement avec influence de la température.

Les imperfections majeures de ces modèles mathématiques sont relatives:

- d'une part, à la prise en compte de la turbulence; c'est un problème qui est encore loin d'être résolu de façon satisfaisante;

- d'autre part, à la représentation géométrique du domaine de calcul dont il a déjà été question plus haut.

C'est la raison pour laquelle les résultats obtenus n'ont pas toujours été pris au pied de la lettre, mais ont plutôt servi de support à l'analyse des phénomènes.

\section{IV.1 - Calcul de la zone d'intercuve sifuée sous le redan}

En se plaçant dans un plan méridien de l'intercuve, on considère le sodium compris entre la cuve primaire (virole cylindrique et redan conique), le baffle du circuit de refiroidissement de la cuve principale et la circulation forcée entre échangeurs et pompes. Cette dernière, n'étani pas située dans le plan du calcul, on a dû la schématiser par un jet rabattu dans ce plan (les deux sens possibles d'écoulement ont été étudiés).

Les conditions aux linnites thermiques sont données par le modèle d'ensemble du réacteur, ainsi que la température du rejet du débit de refroidissement.

On a étudié ainsi deux régimes établis : le régime nominal et un régime partiel pour lequel le débit des pompes est réduit à $17 \%$ du débit nominal, le $\Delta T$ est de $160^{\circ} \mathrm{C}$ comme en régime nominal. Pour chaque régime, les calculs ont été effectués en faisant varier la cote du rejet. Dans tous les cas, la température au-dessus du redan est chaude et le jet des échangeurs est froid.

\section{Résultats}

Ces calculs ont donné deux types de résultats :

- d'une part, les renseignements que l'on en attendait et qui sont liés à la finesse du calcul bidimensionnel;

- d'autre part, des résultats globaux permettant d'étayer la schématisation d'ensemble du bloc-pile.

La figure 6 montre les températures obtenues sur plusieurs verticales, pour deux cotes différentes du rejet (le jet de l'échangeur est au niveau des flèches tracées en bas à gauche).

Dans chacun des cas, les courbes sont en partie confondues, car les isothermes sont pratiquement horizontales, sauf au voisinage immédiat des parois.

On constate que, sous le redan, il existe une zone tampon dont la température est rendue relativement homogène par la convection naturelle qui s'y déverse. En dessous, la température décroît à peu près linéairement, avec une variation de la pente au niveau du rejet du circuit de refroidissement.

Il y a un gradient plus fort, mais assez instable, lorsqu'on atteint le jet de l'échangeur. La variation rapide de température, que l'on remarque à l'extrémité supérieure de chaque courbe, correspond à la traversée de la couche limite qui longe le redan.

Dans l'ensemble des cas étudiés, on a toujours trouvé que la température de peau du redan, côté intercuve, s'élevait doucement le long d'une génératrice (fig. 7) à condition de ne pas placer le rejet trop près de l'arrondi supérieur. Les gradients longitudinaux sont compris entre 0 et $15^{\circ} \mathrm{C} / \mathrm{m}$; ceci écarte la crainte d'avoir un redan soumis à des hétérogénéités de température en partie courante.

En ce qui concerne les enseignements d'ordre général à tirer de ces calculs, citons deux points importants :

$1^{\circ}$ les résultats confirment le découpage par zones adopté dans le calcul global; en effet, on constate que la transition la plus importante, entre sodium chaud et sodium froid dans l'intercuve, est située au niveau du bord supérieur des fenêtres d'échangeurs;

2" le coefficient d'échange, trouvé par le calcul bidimensionnel, entre le redan et le sodium de l'intercuve est du même ordre que celui qu'utilise le calcul global; en effet, la figure 8 donne le flux moyen qui traverse le redan, en fonction de la chute de température dans la couche limite, pour tous les calculs bidimensionnels effectués; on a tracé également la loi de flux adoptée 


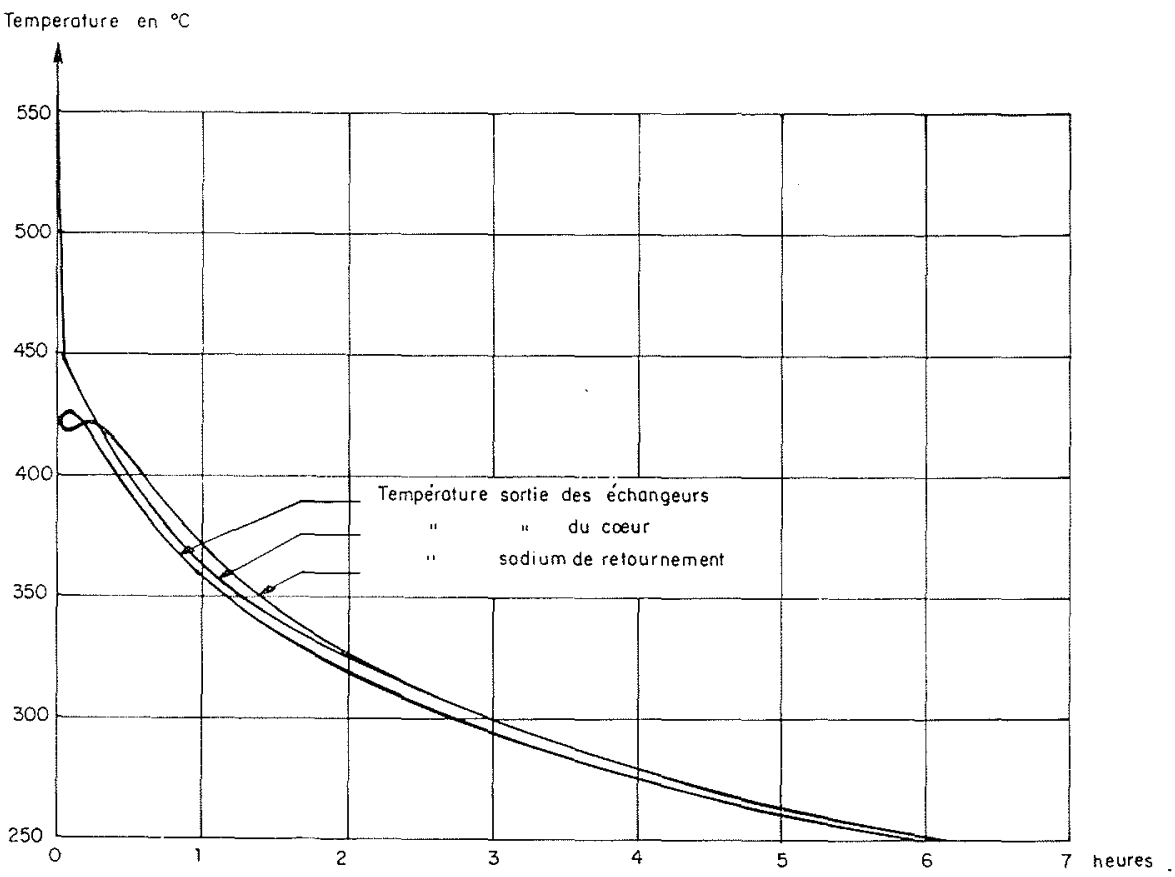

5/ Arrêt d'urgence $C_{1}$ (débit $100 \%$ ).
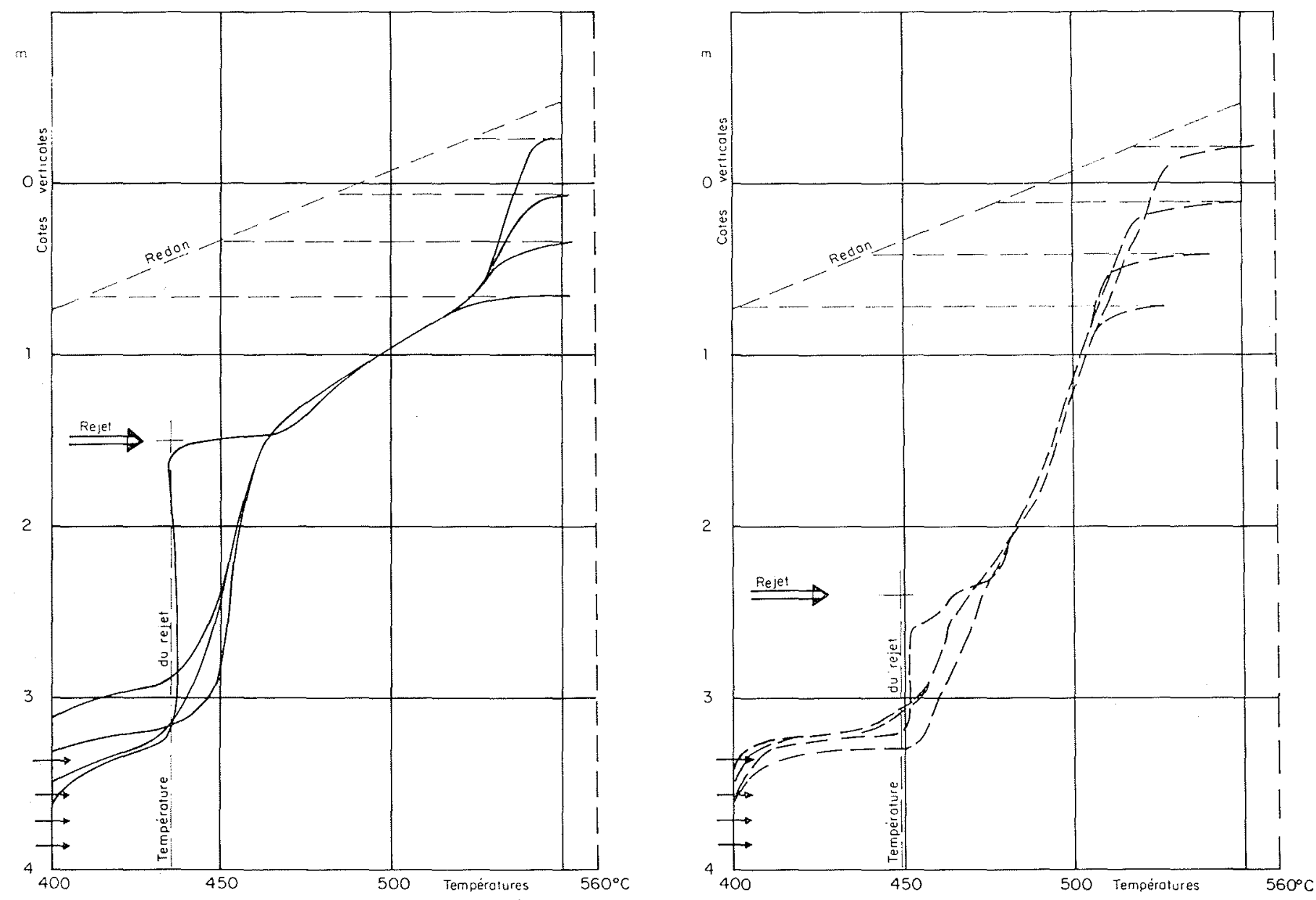

6/ Phénix intercuve. Régime nominal $\left(400-560^{\circ} \mathrm{C}\right)$. 


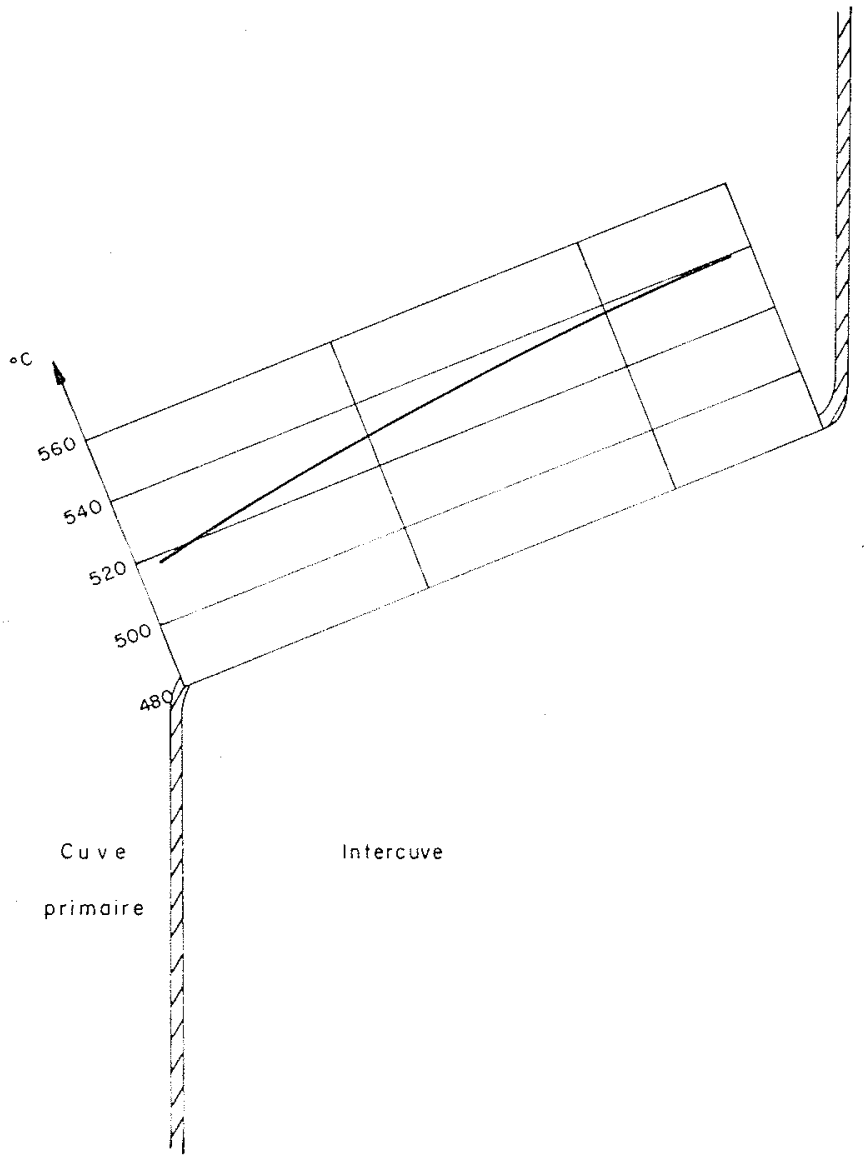

7/ Température du redan côté intercuve.

dans le calcul global; on en déduit que le coefficient d'échange dans le calcul bidimensionnel est:

$$
h=1.2310^{3}\left(\mathrm{~W} / \mathrm{m}^{2}{ }^{\circ} \mathrm{C}\right)
$$

alors qu'on avait :

$h=0.7710^{3} \Delta T^{1 / 3}\left(\mathrm{~W} / \mathrm{m}^{2}{ }^{\circ} \mathrm{C}\right)$ dans le calcul global

c'est-à-dire que le coefficient d'échange du calcul global est un peu plus fort dès que le $\Delta T$ est supérieur à $4^{\circ} \mathrm{C}$.

\section{IV.2 - Calcul du refournement du circuit de refroidissement de la cuve principale}

Ce calcul est effectué, dans un plan méridien de l'espace annulaire, entre la cuve principale et le contre-baffle, en partie haute du circuit de refroidissement.

La température du sodium, au niveau du retournement, est donnée par le calcul global; la cuve principale est supposée adiabatique, tandis que le contre-baffle est le siège d'un flux de chaleur provenant du collecteur chaud.

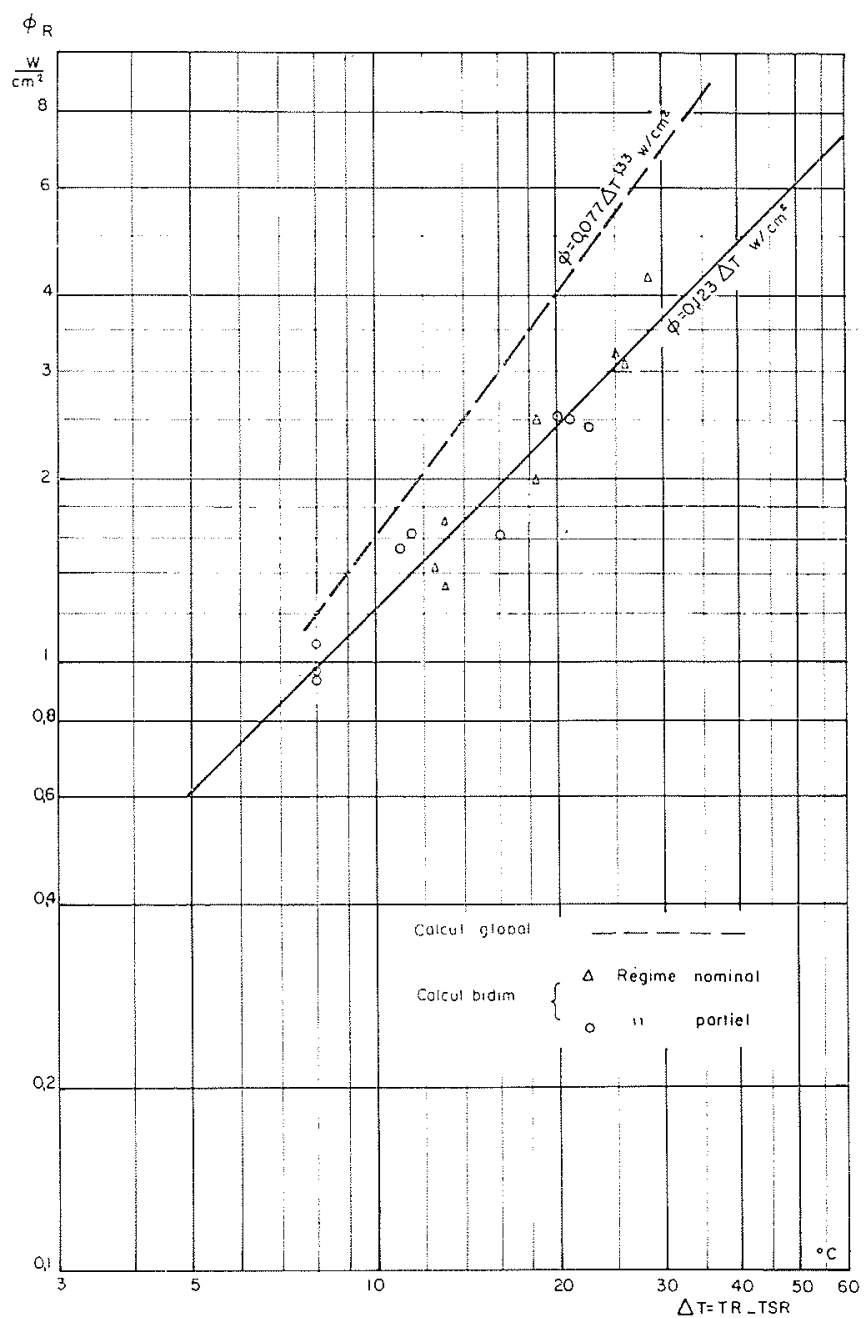

8/ Flux moyen à travers le redan en fonction du $\Delta T$ moyen dans le fluide adjacent.

Sur les figures 9 et 10 on voit, grâce au tracé des lignes de courant de l'écoulement, que le débit se retourne audessus du baffle sur une épaisseur d'environ $5 \mathrm{~cm}$. Les isothermes sont horizontales dans la zone stagnante, sauf au voisinage immédiat du contre-baffle (à gauche sur la figure).

La courbe de température est celle que l'on trouve sur la cuve principale, elle vient compléter la répartition donnée par le calcul global depuis le bas de la cuve.

\section{V - Conclusion}

L'évaluation des températures des cuves des réacteurs à neutrons rapides de type intégré résulte d'un ensemble d'études soit expérimentales, soit théoriques. Compte tenu des intéractions entre les diverses parties du bloc pile, l'outil propre à fournir ces températures est un modèle d'ensemble; pour des raisons de similitude, il s'agit d'un modèle mathématique et non d'une maquette; cependant, il est nécessaire de l'étayer par des résultats expérimentaux guidant la compréhension des phénomènes physiques. Par ailleurs, on a la possibilité d'obtenir un «effet loupe»sur certains points de ce modèle d'ensemble, en utilisant des 
O. DAUBERT et J.-F. MALHERBE

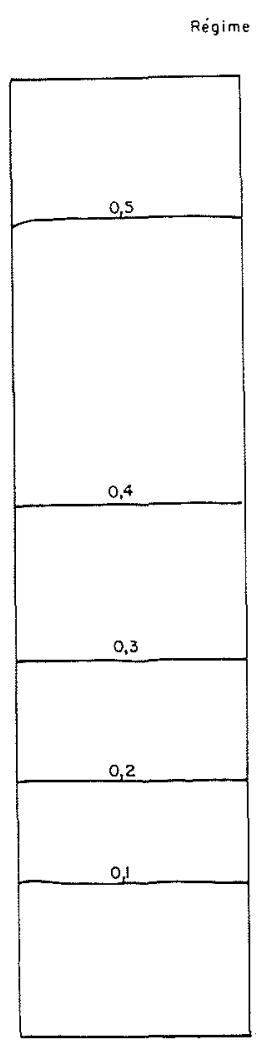

91
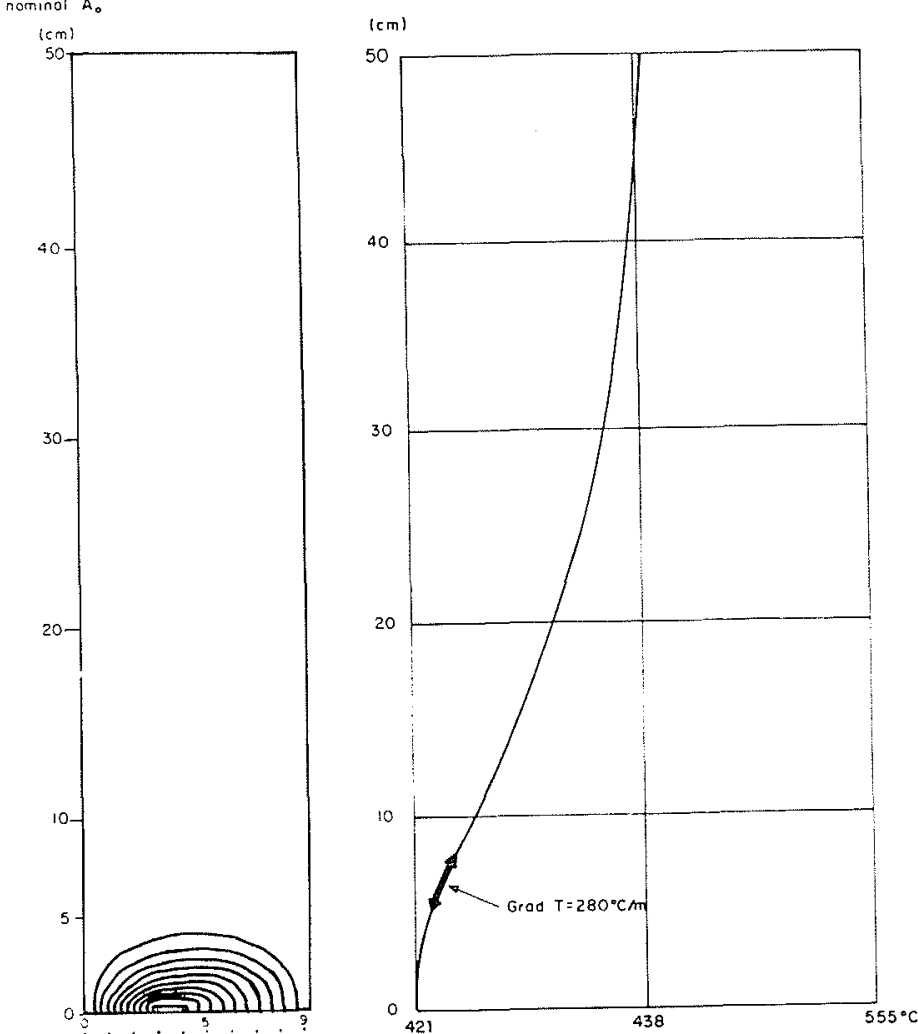

Lignes de couront

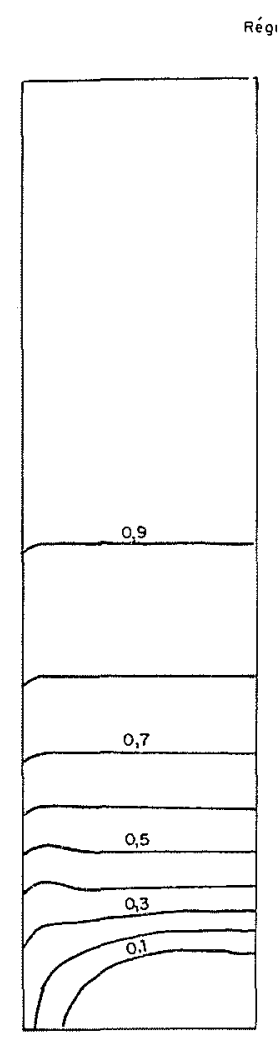

isothermes
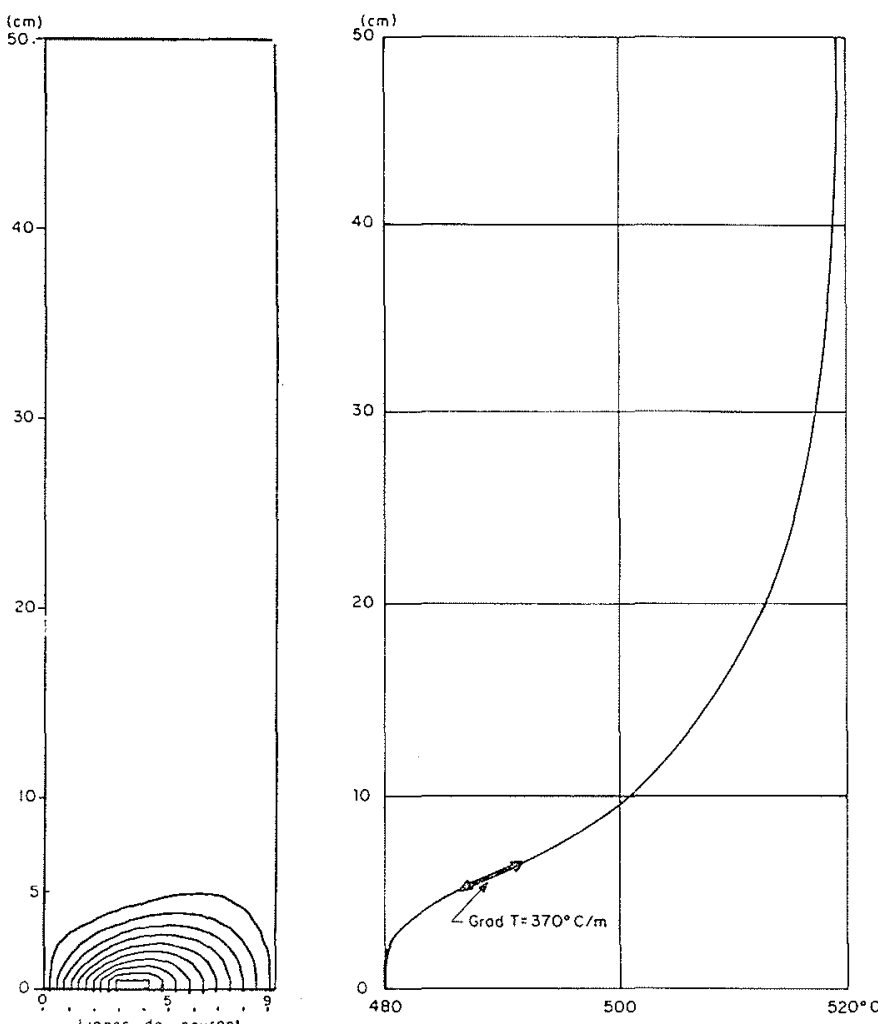
calculs donnant un champ de température, dans un domaine jusqu'ici limité à deux dimensions.

Il est clair que les calculs concernant les réacteurs de la filière vont bénéficier, d'une part des améliorations appontées progressivement aux modèles mathématiques d'écoulement avec influence de la température et, d'autre part, des informations très précieuses que vont fournir les mesures effectuées sur Phénix.

\section{Références}

[1] Daubert (A.) et Huffenus (J.-P.). - Méthodes d'approche pour les problèmes de convection mixte dans les métaux liquides. XIV ${ }^{\text {e }}$ Congrès de l'A.I.R.H., Paris (août-septembre 1973).

[2] Kutatelaze (S.-S.) and al. - Liquid metal heat transfer media. Supplement $\mathrm{n}^{\circ} 2$ of the Soviet Journal of Atomic Energie, Atomic Press, Moscou (1958).

\section{Discussion}

Président : M. H. MONDIN

M. le Président remercie M. MaLherbe pour son intéressant exposé et ouvre la discussion.

M. GAUTHIER voudrait savoir si les modèles mathématiques de simulation décrits prenaient en compte l'influence de la turbulence de l'écoulement (en utilisant, par exemple la méthode proposée par M. HuFfenus de Grenoble).

Nous avons, répond M. MALHerbe, supposé constants les coefficients de diffusivité de quantité de mouvement et de chaleur en les assimilant à des coefficients de diffusion moléculaire. Nous avons jugé que les problèmes de simulation de turbulence étaient encore loin d'être résolus de façon satisfaisante. Nous avons été en contact avec M. Huffenus à ce sujet ; les résultats obtenus par lui étaient d'ailleurs peu différents de ceux que nous avons calculés.

Afin d'éclairer les problèmes posés par «l'intercuve», M. DAuberI résume brièvement, en ces termes, l'évolution des études et essais auxquels ils ont donné lieu :

Toute l'équipe qui a travaillé sur le projet Phénix s'est, dès l'origine, intéressée aux phénomènes observés au ras du bord supélieur des fenêtres de sortie des échangeurs.

Au début, nous redoutions à ce niveau - et toutes les expériences semblaient plutôt indiquer que cette crainte était justifiée - des écarts de température de $160^{\circ} \mathrm{C}$ sur une épaisseur très faible. Or, vous avez pu voir sur le dessin projeté, qu'ils ont été abaissés vers $50^{\circ} \mathrm{C}$; c'est un progrès énorme par rapport aux 150 ou $160^{\circ} \mathrm{C}$ que l'on craignait au départ.

A quoi est due la diminution considérable de ce gradient au cours de l'évolution du projet Phénix?

Au départ, nous avions des fuites à la traversée des échangeurs. Elles apportaient dans cette zone de l'intercuve, un nombre important de calories, directement par convection forcée.

Des expériences ont été menées à Cadarache (et reprises au C.E.N.G.) en eau chaude. Elles étaient relativement difficiles puisqu'il fallait contrôler les conditions aux limites thermiques.

Les expériences que nous avons entreprises à Chatou, en eau salée, ont montré que pratiquement l'intercuve était entièrement chaude tant qu'il y avait aux échangeurs la fuite thermique susdite.

Donc, le premier progrès important, à mon avis, a été l'introduction du joint par siphon cassé qui interdit tout passage dans l'intercuve du sodium chaud venant de la cuve interne. Il serait trop long d'expliquer ici pourquoi une convection forcée entraîne effectivement un gradient de température obligatoirement important.

Ensuite, ainsi que l'a expliqué $M$. SAUvage, ce qui a fait progresser d'un cran de plus le problème de l'intercuve est l'évolution de la température de la cuve principale, imposée par les métallurgistes. Ceci conduisit à ce qui a été appelé «la cuve tiède ».

La fuite aux échangeurs, qui était très dangereuse en raison de sa température élevée, n'a plus ici la même infuence parce qu'on dispose d'une «fuite froide» provenant des baffles. Le même effet de la convection est utilisé, mais avec une température plus basse, d'où une diminution du gradient au-dessus de la sortie des échangeurs.

En ce qui concerne les modèles de turbulence, je dois dire que nous avons, de notre côté, fait des recherches. Nous les continuons d'ailleurs en faisant une simulation de la turbulence dans un jet en milieu confiné.

En même temps, la SOGREAH avait fait des études pour comparer les résultats avec d'autres calculs que nous ne considérions pas comme assez développés chez nous, à Chatou, pour être sûrs de la simulation de la turbulence. On a done comparé la simulation de M. Huffenus à la nôtre :' je crois que les résultats étaient très voisins en ce qui concerne les températures obtenues.

Dans cette autre simulation, ce n'est pas la prise en compte de la turbulence qui avait permis de déterminer le gradient que vous avez souligné, car il avait été pratiquement donné par un coefficient d'échange imposé à la limite des jets des échangeurs. On avait notamment discuté de ce coefficient avec M. HuFfenus, de façon à essayer de représenter aussi fidèlement que possible les phénomènes.

Le but de l'aménagement de l'intercuve était aussi de conserver, sous le redan, une seconde zone qui devait être plus chaude, afin de protéger le redan contre un gradient transversal - et également longitudinal - important; on voulait, de plus, qu'il n'y ait pas de transition à fort gradient.

Ceci s'est fait, presque naturellement, par la convection naturelle en milieu confiné, puisque celle-ci amène dı sodium chaud qui s'accumule dans la partie supérieure. La nature même du métal liquide a favorisé aussi les choses car, non seulement les métaux liquides son bons conducteurs, mais, en outre, ils ont un nombre de Prandtl qui est faible en raison de leur basse viscosité; ainsi le sodium placé sous le redan n'est pas trop entrâné par viscosité.

M. le Professeur ForTier demande quelles sont les conditions aux limites «thermiques» adoptées dans le modèle bidimensionnel.

On a vu, répond M. Matherbe, que les zones sur lesquelles portaient les calculs bidimensionnels étaient entourées par des zones de convection forcée, importante (comme dans le collecteur chaud) ou à faible vitesse (comme à l'intérieur des baffles). De l'autre côté des parois de la zone calculée, on connaît donc les températures par le modèle global du bloc-pile. On impose alors des conditions aux limites mixtes, faisant intervenir ces températures et la résistance thermique de la paroi

M. le Président remercie les personnes qui ont animé la discussion et donne la parole à $M$. Grand pour l'exposé de la communication suivante; celle-ci est illustrée par la présentation d'un remarquable film montrant la visualisation, par la méthode de strioscopie en couleur, de l'évolution des courants de circulation suivant lo nombre de Reynolds et le nombre de Grashof. 